\title{
Q-Initiativen - Online-Plattform der FMH für Qualität in der Medizin
}

Seit 2007 dokumentiert die FMH Qualitätsaktivitäten auf ihrer Website (www.fmh.ch $\rightarrow$ Qualität $\rightarrow$ Qualitätsinitiativen). Die Verbindung der Schweizer Ärztinnen und Ärzte hat damit eine Plattform für Wissensvermittlung und Vernetzung von Qualitätsbestrebungen in der Schweiz geschaffen. Diese Plattform zeigt die Bandbreite der medizinischen Qualitätssicherung und -entwicklung in der Schweiz auf.

Aufgenommen werden Qualitätsinitiativen, bei denen es sich um eine Massnahme zur Sicherung und Verbesserung der Qualität der ärztlichen Leistung handelt. Zudem sollen die Qualitätsaktivitäten Prozesse verbessern und Fehler reduzieren, was sich positiv auf die Patientensicherheit und die Behandlung auswirken kann. Initianten von Q-Initiativen können Einzelpersonen, Netzwerke oder Institutionen sein, die nachweisbar Qualitätsentwicklung betreiben. Die Bandbreite der Q-Initiativen reicht von Kleinprojekten bis hin zu etablierten Qualitätsmanagementsystemen.

\section{Per Suchmaschine sofort beim richtigen Projekt}

Die Online-Porträts der vorgestellten Qualitätsinitiativen enthalten einen Kurzbeschrieb sowie weitere Informationen wie Ablauf, Zeitaufwand, Kosten und Kontaktdaten. Mit einer gezielten Suche nach Fach, Praxisgrösse, Arbeitsbereich (ambulant/stationär) und Sparte (Akutspital, Rehabilitation, Langzeitpflege usw.) lassen sich rasch all jene Qualitätsinitiativen finden, die den Bedürfnissen des Nutzers entsprechen.

\section{Aufnahme von neuen Qualitätsinitiativen}

Nach wie vor gibt es viele Qualitätsinitiativen, die noch nicht auf der FMH-Website präsent sind und einem breiten Fachpublikum vorgestellt werden sollten. Nutzen Sie die Gelegenheit, und melden Sie Ihre Qualitätsaktivitäten für die Aufnahme auf der OnlinePlattform für Qualitätsinitiativen in der Medizin der FMH an!

Alle neuen Q-Initiativen, die bis zum 5. März 2012 bei der Abteilung Daten, Demographie und Qualität DDQ der FMH eingegangen sind, beurteilt die Arbeitsgruppe Qualität AGQ-FMH anhand von Kriterien zur Durchführbarkeit, Übertragbarkeit, zum Nutzen und Gesamteindruck usw. Nach der Veröffentlichung auf der Online-Plattform publiziert die FMH die zwölf geeignetsten Q-Initiativen wie bisher im Rahmen einer Kleinporträt-Serie in der Schweizerischen Ärztezeitung. Weiter werden einzelne Q-Initianten eingeladen, ihre Projekte der Arbeitsgruppe Qualität der FMH und der Abteilung Daten, Demographie und Qualität (DDQ) zu präsentieren.

\section{Möchten Sie Ihre Qualitätsinitiative auf der Online-Plattform der FMH publizieren?} Und später möglicherweise auch in der Schweizerischen Ärztezeitung präsentieren? Kontaktieren Sie uns über ddq[at]fmh.ch oder 031 35911 11, wir informieren Sie gerne detailliert über das weitere Vorgehen. Anmeldeschluss ist der 5. März 2012.

\section{Bringt Vorteile. Die Mitgliedschaft in der FMH.}

Wir machen uns stark für Sie. Mit engagierter Standespolitik und attraktiven Services: TarifInfoline, Rechtsauskünfte, Musterverträge.

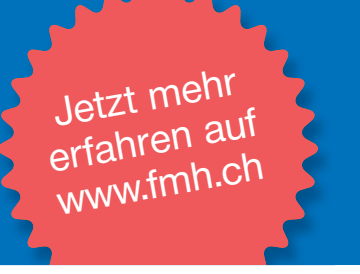

